\title{
Serie de Violencias y Contestaciones en la Producción del Espacio Urbano Periférico del Ecuador
}

\begin{abstract}
Autores: Gustavo Durán, Manuel Bayón, Alejandra Bonilla y Michael Janoschka. ISBN: 9789942381910 (32 páginas), 9789942381835 (34 páginas), 9789942381842 (42 páginas), 9789942381859 (33 páginas), 9789942381866 (37 páginas), 9789942381873 (25 páginas), 9789942381880 (31 páginas), 9789942381897 (38 páginas), 9789942381903 (37 páginas).

Esta colección presenta la investigación de un año sobre problemáticas urbanas poco tratadas en la literatura del Ecuador en materia de desarrollo de sus ciudades desde el punto de vista de sus espacios periféricos. En las dos últimas décadas se ha dado un fuerte crecimiento de las ciudades en Ecuador, apareciendo una ingente cantidad de nuevos núcleos urbanos, y consolidándose nuevas ciudades intermedias y redes urbanas, que han tenido una escasa atención por parte de los estudios urbanos en el país, centrándose la amplia mayoría de casos de estudio en Quito, Guayaquil y Cuenca en este orden, con espacios y casos de estas ciudades muy predominantes.

La serie se compone de 8 publicaciones sobre casos de 8 diferentes ciudades del Ecuador, y una cartilla de síntesis y recomendaciones de política pública. Cada uno de los estudios de caso, contemplan un mismo corpus teórico en relación con la producción del espacio urbano periférico a través de una dialéctica de violencias y contestaciones que generan diferentes resultados en función del tipo de capital predominante en relación a las diferentes ciudades analizadas, la actuación del Estado en sus diferentes niveles y las formas de contestación por parte de los distintos sujetos analizados.

Estos casos de estudio se centran en Ciudad Bicentenario de Quito, Monte Sinaí en Guayaquil, la reconstrucción y El Guabito en Portoviejo, la Ribera en Esmeraldas, el sur de Santo Domingo de los Tsáchilas, el barrio 25 de febrero en Lago Agrio, el Mirador en Puerto Ayora y Tundayme en El Pangui. Estos 8 casos son una muestra de la forma en la que se han producido las periferias urbanas
\end{abstract}


en el país, con casos muy diversos: la política de vivienda social, la renovación urbana mediante desalojos, los modelos urbanos post-desastre del terremoto en Manabí, la implementación del modelo turístico urbano, la urbanización de áreas agroindustriales o los nuevos barrios alrededor de la actividad petrolera, minera o turística.

Las violencias transitan desde las más contundentes, como el desalojo policial y destrucción de la vivienda en pocos minutos que vivieron las familias en Guayaquil y El Pangui, violencias más cotidianas asociadas a la contaminación por actividades rentistas en Santo Domingo o Lago Agrio, las políticas de desplazamiento de los negocios populares en los planes de turistificación en Esmeraldas o Portoviejo, o las violencias asociadas a la forma en el que transitan las familias hacia las Viviendas de Interés Social en Quito o Guayaquil. Dos acciones de alrededor del megaplan de vivienda Ciudad Bicentenario de Quito ilustran bien las formas de contestación recogidas en esta serie: el mismo día que boicoteaban las obras del Alcalde Mauricio Rodas porque no entregaban la vivienda a las familias beneficiarias, estaban supervisando materiales y espacios verdes en sus futuras viviendas. Es decir, la investigación y los casos recogen una dimensión de contestación más visible en el que las protestas o negociaciones son fundamentales, pero también una dimensión más cotidiana de construcción familiar y colectiva del espacio de vida.

Sin embargo, la serie busca salir de ahondar hasta el infinito en cada uno de los casos, sino que trata de abstraer la forma en la que se producen el espacio periférico urbano del Ecuador. Los aprendizajes de estos 8 casos están articulados en tres ejes de aprendizajes de la interacción entre violencias y contestaciones en la producción del espacio urbano periférico: el modelo de Vivienda de Interés Social en Quito, Guayaquily Portoviejo; la implementación de las renovaciones urbanas amparadas en la atracción de turismo en Esmeraldas, Portoviejo y Puerto Ayora; y las nuevas periferias generadas alrededor de actividades extractivas en Lago Agrio, Santo Domingo y El Pangui. El primer eje de Vivienda de Interés Social es sobre el que se presentan unos aprendizajes más nítidos, mientras que en los ejes de renovación urbana turística y nuevas periferias turísticas la reflexión es más incipiente y novedosa. Estos tres ejes muestran las principales formas de configuración de periferias urbanas con diferentes actores, pero con tendencias comunes generales.

La serie logra no solamente generar una discusión teórica, sino que se aventura también en la intervención en las políticas con recomendaciones. El documento final llamado "Cuadernillo síntesis y recomendaciones de política pública" recoge de forma multiescalar este diálogo de violencias y contestaciones en la producción de nuevas periferias mediante el modelo de Vivienda de Interés Social. A través de la escala de la vivienda, el barrio y la ciudad, y en una contundente explicación de las formas de violencia y contestación que se han dado en cada una con profusos ejemplos de los casos analizados en Quito, 
Guayaquil y Portoviejo, el documento genera una serie de recomendaciones nítidas para cada una de las problemáticas analizadas.

Algunas de las más destacadas recomendaciones son considerar las esferas productivas y reproductivas con materiales durables en el diseño de viviendas, detener las políticas de desalojos, crear mecanismos para que las cooperativas sean protagonistas en la producción del espacio, considerar los servicios y el hábitat como parte indisociable de las viviendas, así como la densificación de los vacíos urbanos dedicados a la especulación con programas de vivienda en las áreas centrales pensados en las familias usuarias y no en los grandes constructores. No se tratan de recomendaciones que hayan estado fuera del debate académico y social sobre la cuestión. Lo novedoso se encuentra en la sistematización por escalas de estas recomendaciones en relación a los casos estudiados, y la propuesta de puesta en marcha de programas concretos para generar una nueva forma de afrontar la provisión de vivienda a los sectores populares desde el Estado.

Estas recomendaciones llegan en medio del comienzo del debate en la Asamblea Nacional de la nueva Ley de Vivienda Social desde el mes de mayo alrededor de las medidas económicas para enfrentar la crisis de la pandemia COVID-19. En la propuesta de los asambleístas se encuentran grandes promociones de vivienda, poniendo el acento en la necesidad de reactivar el sector de la construcción, más que en las necesidades de las familias usuarias, con megaplanes allá donde el valor de los suelos sea más barato y promoviendo economías de escala con la ejecución simultánea de miles de viviendas. Desde los aprendizajes de Contested Cities Ecuador se plantea lo contrario: la promoción de pequeñas intervenciones en espacios consolidados de la ciudad, donde se priorice la Producción Social del Hábitat, para que las familias usuarios no sean el cupo para reactivar un sector económico, sino sujetos de la construcción de su espacio de vida, con derecho a viviendas de calidad, en entornos habitables, y cercanos a los centros de empleo y servicios.

Otro eje importante en esta serie es la participación de equipos de las ciudades investigadas en Esmeraldas, Portoviejo, Guayaquil y El Pangui, lo que permite una visión aterrizada en las realidades locales. La participación de las dirigencias barriales y comunitarias de los espacios analizados ha permitido una rápida socialización y que los documentos elaborados por la investigación, además de ser una forma de visibilización y análisis, se promueven también como una herramienta de transformación de las realidades observadas. Las publicaciones proponen así un relato que emana de los sujetos, rompiendo así las concepciones preconcebidas y en muchas ocasiones estigmatizantes que las élites estatales o corporativas tienen sobre las poblaciones receptoras de las políticas de planificación que son analizadas.

La "Serie de Violencias Y Contestaciones En La Producción Del Espacio Urbano Periférico Del Ecuador" no tiene su mérito en hacer una recopilación totalizante del estado de la cuestión urbana en el país, sino en trazar una nueva 
geografía crítica de la urbanización. Esta perspectiva se centra en espacios poco tratados desde la perspectiva de los estudios urbanos, con novedosos ejes de comparación que profundicen en los aspectos más ocultados del nuevo patrón de urbanización, y con una vocación de intervención útil en las políticas públicas, poniendo énfasis en la participación de los sectores populares relacionados con las mismas. 